\title{
3D-measurement with the stereo scanning electron microscope on sub-micrometer structures
}

\section{T. Vynnyk}

taras.vynnyk@imr.uni-hannover.de

\section{T. Schultheis}

In this paper the photometric or the so called "shape from shading" method is presented. In comparison to existing methods the efficiency of the detector system was considered and the requirements of the cosine Lambert's law for the angle distribution of the emitted electrons are suppressed. This new method was experimentally verified by measuring a steel sphere, a holographic grating and a hologram. [DOI: 10.2971/jeos.2010.10038s]

Keywords: stereo electron microscope (SEM), 3D-surface reconstruction, shape from shading, holographic diffraction grating, hologram

\section{INTRODUCTION}

Since almost 30 years attempts have been made to change the SEM in the sub-micron range into a $3 \mathrm{D}$ measuring device. There exist different approaches, among them the "shape from shading" method. The basics of the photometric method were developed in the 90's [1]-[5]. The method uses the assumption that the emission yield $\sigma(\phi)$ depends on the local inclination angle of the specimen $\phi$ as: $\sigma(\phi)=\sigma_{0} / \cos \phi$. Furthermore the angle distribution of the emitted electrons should follow the Lambert cosine law: $f(\psi)=\cos \psi$. If a symmetrical 2-detector system is used for registration of the emitted electrons [6] (see Figure 1) then the detector signals are: $I_{1,2}=(1 / 2) \cdot \sigma(\phi) \cdot(1 \mp \sin \phi)$ and therefore the difference is: $I_{2}-I_{1}=\sigma_{0} \tan \phi=\sigma_{0} \frac{d z(x)}{d x}$. Finally the surface is obtained via numeric integration of the partial derivatives.

Despite the simple reconstruction algorithm, the photometric method was not established as standard measuring method due to the following reasons. The material dependent emission coefficient $\sigma$ in Eq. (1) is unknown. The photometric method assumes that the emission yield behaves as $1 / \cos$ of the local inclination angle of the measured specimen. This assumption is correct only for primary electrons with the energy $>10 \mathrm{keV}$ (see Figure 2(a)). However, the use of such primary electrons increases both the BSE - fraction and the emission volume (electron range, see Figure 2(b)), which leads to loss of the lateral resolution. Besides, the angular distribution of BSE strongly depends on the incident angle of the primary electron beam. The angular distribution of the secondary emission electrons (SE) is assumed to have a cosine form. This assumption is a very rough approximation of the real one. The

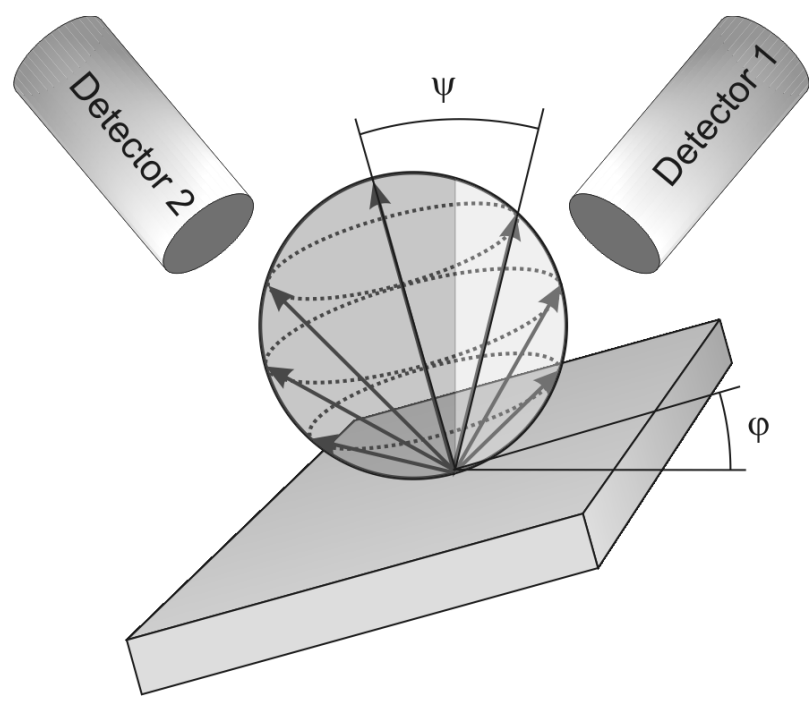

FIG. 1 2-detector system

alternative approach refers to quantum mechanics and gives a more realistic description of its behaviour [7].

The efficiency factor of the detector system is supposed to be one. In reality the efficiency is always smaller due to the influence of the specimen chamber and of the electron gun. According to the recent publications the efficiency of one ET Detector lies approximately about $30 \%-40 \%[8]$.

In this paper a new mathematical model of the detector signal occurrence is presented. This model provides the basis for the algorithm of the surface reconstruction, which is experimen- 

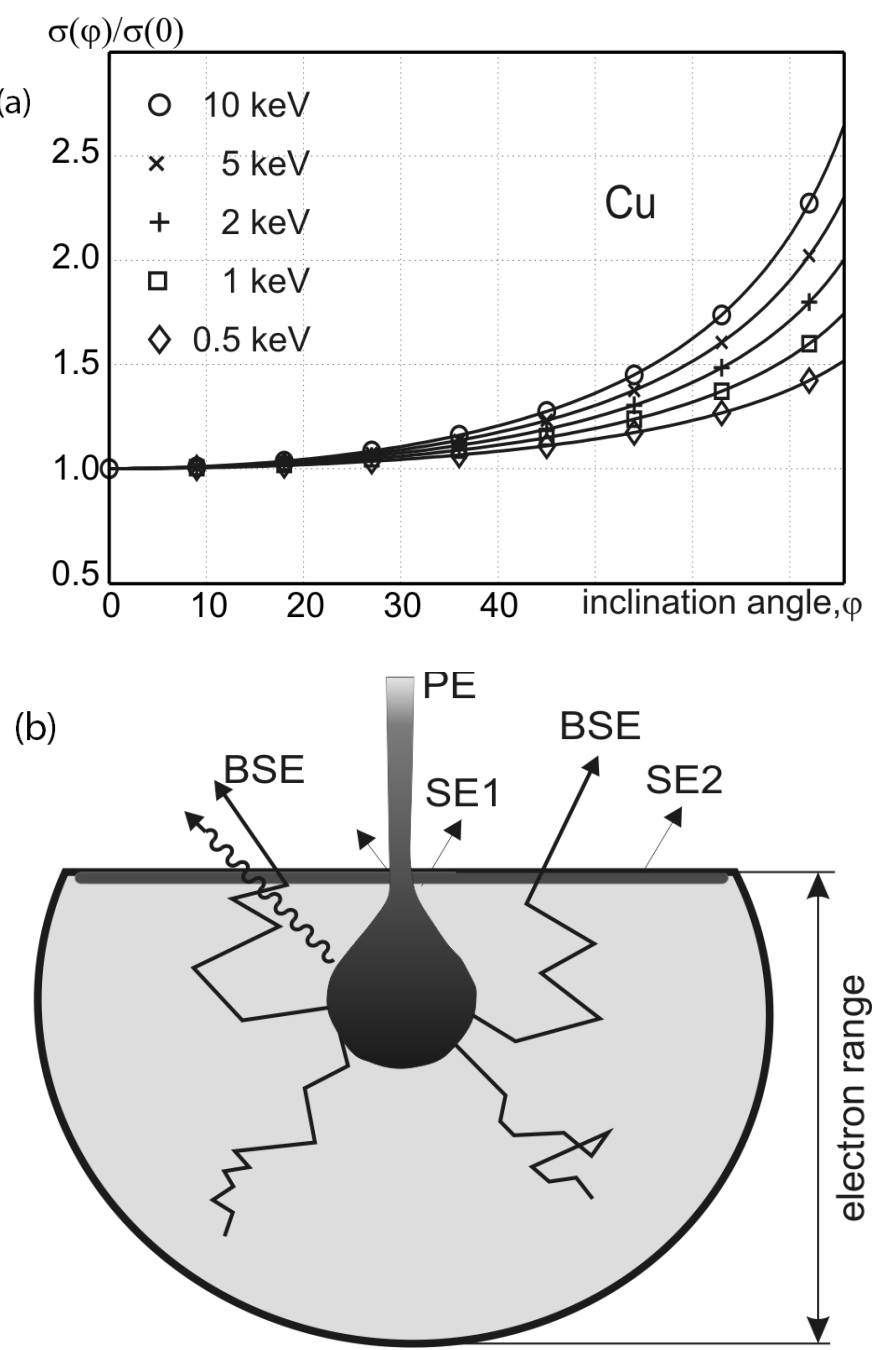

FIC. 2 (a) Dependence of the emission yield on the inclination angle and (b) occurrence of the secondary electrons.

tally verified on holographic gratings. The main advantage of this model over former methods is, that it takes into account the efficiency of the detector system as well as it improves assumptions concerning properties of the emitted electrons. The conventional SEM was additionally equipped with two vis-à-vis positioned Everhardt-Thornley (ET) detectors and a Z-rotation unit (see Figure 3).

The rotation of the sample allows to position the ET detectors first along the x-axis and then along the y-axis to record the $I_{1 x^{-}}, I_{2 x^{-}}, I_{1 y^{-}}$and $I_{2 y}$-signals. These signals are functionally connected with the partial derivatives of the sample surface which provide the basis of the surface reconstruction:

$$
\begin{aligned}
& \frac{I_{1 x}-I_{2 x}}{I_{1 x}+I_{2 x}}=F x\left(z(x, y), \frac{\partial z}{\partial x}, \frac{\partial z}{\partial y}\right) \\
& \frac{I_{1 y}-I_{2 y}}{I_{1 y}+I_{2 y}}=F y\left(z(x, y), \frac{\partial z}{\partial x}, \frac{\partial z}{\partial y}\right) .
\end{aligned}
$$

\section{DISCUSSION}

The reconstruction formulas are based on the following assumptions. The angle distribution takes the following form:

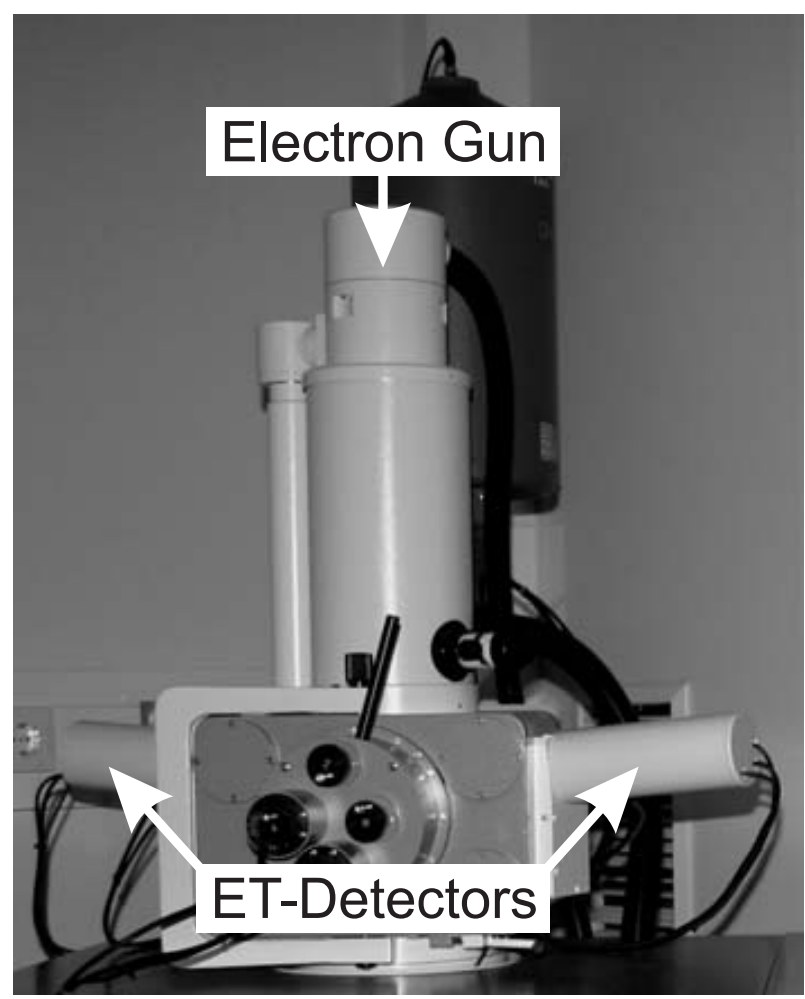

FIG. 3 SEM with 2 ET-detectors.

$$
f(\psi)=\frac{1}{\pi}\left(k_{1} \cos \psi-k_{2} \cos ^{2} \psi\right)
$$

where $\psi$ is the angle between the direction of the emitted electron and the normal to the local tangent plane to the surface in the measured position. The coefficients $k_{1}$ and $k_{2}$ are determined via parameter optimization on experimental data.

The emitted electrons are registered either by one of the 2 detectors or are captured by the surface or electron gun [7]. Based on the FEM simulation results the emission directions were split in 3 zones. Zone 3 describes electrons, which are partly absorbed from the electron gun. Zone 1 stands for the electrons registered by the detectors. Zone 2 represents electrons that are captured theoretically neither by the detectors nor by the electron gun. Nevertheless they are considered as being registered to $50 \%$ by detector 1 and $50 \%$ by detector 2 . To describe this zone splitting two angle parameters are introduced. The first parameter corresponds to half of the opening angle of the electron gun $\theta$, which describes its partial absorption. The second parameter describes the maximum azimuthal angle, at which the emission electrons are registered by the respective detector (see Figure 4(a)).

\section{ZONE 1}

In order to temporally eliminate the influence of the electron gun the $\theta$ angle will be set to $0^{\circ}$. Since this marginal $\left(\theta=0^{\circ}\right)$ case is used for further considerations, Zone 1 here is defined as $Z_{0 n e_{1} 0^{\circ}}$. The electron flow via elementary surface element 

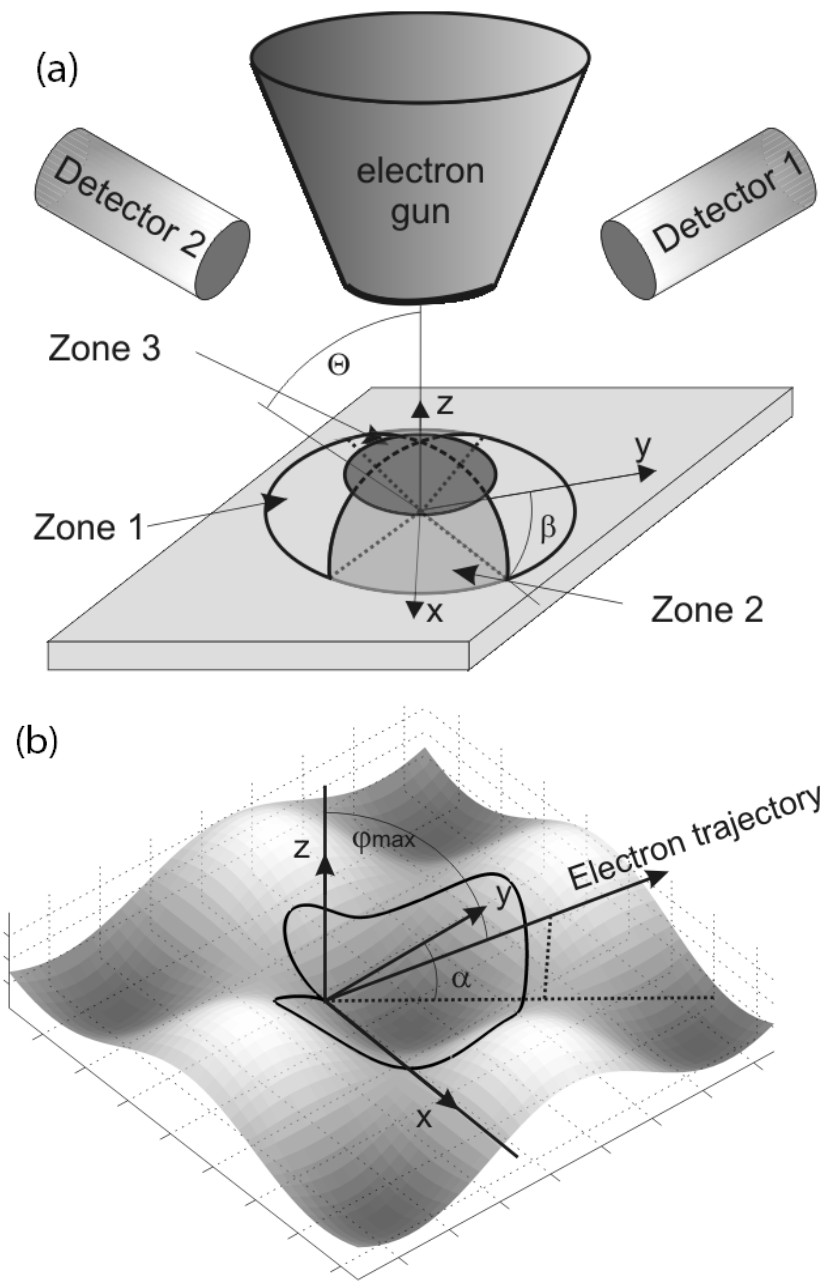

FIG. 4 (a) Zone splitting of the emitted electrons and (b) unregisterable emission fraction.

$d G=r^{2} d \Omega$, (see Figure 5), is:

$$
\begin{aligned}
d \Phi & =\sigma(x, y) f(\gamma, \alpha) r^{-2} d G \\
& =\sigma(x, y)\left(k_{1} f_{\text {Lam }}(\gamma, \alpha)-k_{2} f_{\text {Lam }}^{2}(\gamma, \alpha)\right) d \Omega
\end{aligned}
$$

where $d \Omega$ is a differential solid angle, $r=1$ is the radius of the unit sphere and $f(\gamma, \alpha)$ is the angular distribution of the emitted electrons in the global spherical coordinate system. This distribution depends on zenith angle $\gamma$ and azimuth angle $\alpha$. As already mentioned in Eq. (2), the angular distribution is a superposition of the Lambert's (cosine) $f_{\text {Lam }}(\gamma, \alpha)$ and square Lambert's $f_{\text {Lam }}^{2}(\gamma, \alpha)$ functions. For arbitrary direction $\vec{r}(\gamma, \alpha)=[\sin \gamma \sin \alpha, \sin \gamma \cos \alpha, \cos \gamma]$ the Lambert's distribution takes the following form:

$$
\begin{aligned}
f_{\text {Lam }}(\gamma, \alpha)= & \cos (\vec{r}, \vec{n}) \\
= & \frac{-1}{\pi}\left(\sqrt{1+\frac{\partial z^{2}}{\partial x}+\frac{\partial z^{2}}{\partial y}}\right)^{-1} \\
& \times\left(\frac{\partial z}{\partial x} \sin \gamma \sin \alpha+\frac{\partial z}{\partial y} \sin \gamma \cos \alpha-\cos \gamma\right)
\end{aligned}
$$

Here $\vec{n}$ is the normal to the tangent plane of the surface at the irradiation point: $\vec{n}=\left[-\frac{\partial z}{\partial x},-\frac{\partial z}{\partial y}, 1\right]^{T}$

The signals on the detectors 1 and 2 are proportional to the

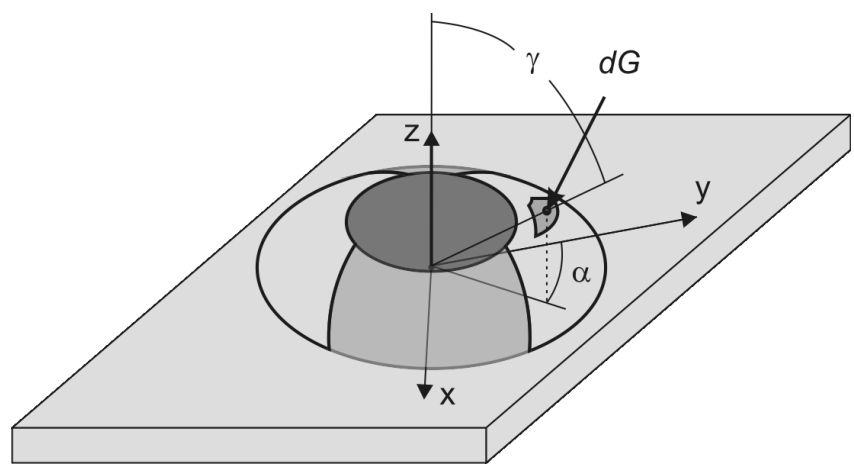

FIG. 5 Electron flow over the elementary surface

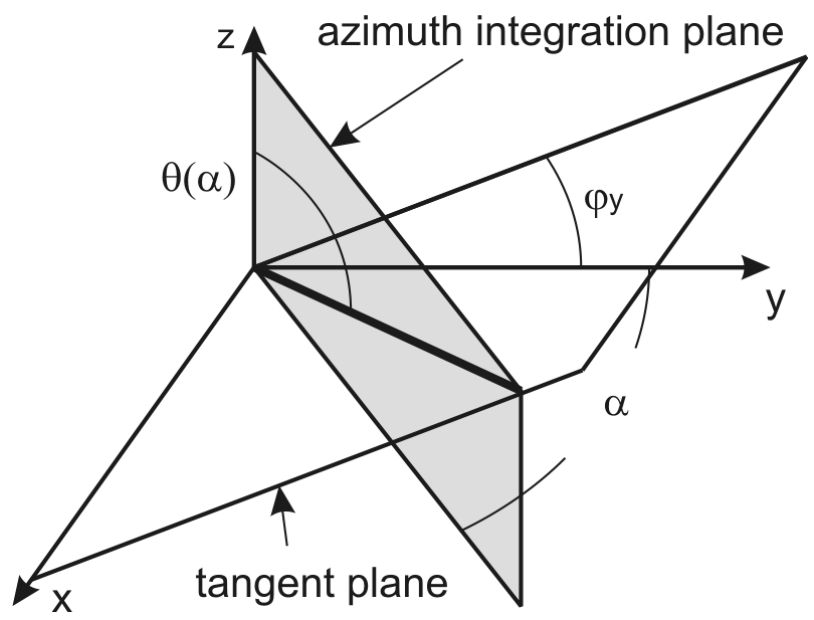

FIG. 6 Evaluation of the upper integration limit.

electron flow $\Phi$ and can be calculated as surface integrals:

$$
\begin{aligned}
\frac{I_{1 y, 2 y}}{\sigma(x, y)}= & \int_{\Omega_{1,2}} f(\gamma, \alpha) d \Omega \\
= & k_{1} \underbrace{\theta(\alpha)}_{\underbrace{}_{a} \int_{\operatorname{Int}_{11}(a, b)}^{b} d \alpha \int_{\operatorname{Lam}}^{\theta(\alpha)}(\gamma, \alpha) \sin \gamma d \gamma} \\
& -k_{2}^{\int_{a}^{b} d \alpha \int_{0}^{b} f_{\operatorname{Lam}^{2}}(\gamma, \alpha) \sin \gamma d \gamma}
\end{aligned}
$$

With $\sigma(x, y)$ as the total emission yield and $\Omega_{1,2}$ as integration domains for the detectors 1 and 2 . In the Cartesian coordinate system these signals can be represented as double integrals over the domain presented via azimuth range $[a, b]=[-\beta, \beta]$ and $[a, b]=[\pi-\beta, \pi+\beta]$ for detectors 1 and 2 respectively and zenith range $[0 \ldots \theta(\alpha)]$. The upper integration limit $\theta(\alpha)$ is defined as the intersection of the tangent plane and the azimuth integration plane $x \cos \alpha-y \sin \alpha=0$ (see Figure 6):

$$
\begin{aligned}
\theta(\alpha) & =\arccos \left(M\left(\sqrt{1+M^{2}}\right)^{-1}\right), \\
M & =\sin \alpha \frac{\partial z}{\partial x}+\cos \alpha \frac{\partial z}{\partial y}
\end{aligned}
$$


After substitution using Eqs. (4) and (6) and simplification the integral $\operatorname{Int}_{11}(a, b)$, Eq. (5) takes the following form:

$$
\begin{aligned}
\operatorname{Int}_{11}(a, b)= & \frac{1}{2 \pi}\left(\sqrt{1+{\frac{\partial z^{2}}{\partial x}}^{2}+\frac{\partial z^{2}}{\partial y}}\right)^{-1} \\
& \times \int_{a}^{b}\left(1-\left(\sin \alpha \frac{\partial z}{\partial x}+\cos \alpha \frac{\partial z}{\partial y}\right) \theta(\alpha)\right) d \alpha
\end{aligned}
$$

Unfortunately this integral cannot be solved via elementary functions. However, for the surface reconstruction using only Zone $_{1 \_0^{\circ}}$, it is not the signal that is significant but their difference:

$$
\begin{aligned}
\frac{I_{2 y}-I_{1 y}}{\sigma(x, y)}= & k_{1} \underbrace{\left(\operatorname{Int}_{11}(\pi-\beta, \pi+\beta)-\operatorname{Int}_{11}(-\beta, \beta)\right)}_{\Delta \operatorname{Int}_{11}} \\
& -k_{2} \underbrace{\left(\operatorname{Int}_{12}(\pi-\beta, \pi+\beta)-\operatorname{Int}_{12}(-\beta, \beta)\right)}_{\Delta \operatorname{Int}_{12}} .
\end{aligned}
$$

The use of relations $\cos (\alpha+\pi)=-\cos \alpha, \sin (\alpha+\pi)=$ $-\sin \alpha, \theta(\alpha+\pi)=\pi-\theta(\alpha)$ leads to:

$$
\Delta \operatorname{Int}_{11}=\sin \beta \sin \varphi_{y}
$$

whereas the inclination angles $\varphi_{x}, \varphi_{y}$ are defined as:

$$
\begin{aligned}
& \sin \varphi_{y}=\frac{\partial z}{\partial y}\left(\sqrt{1+\frac{\partial z^{2}}{\partial x}+\frac{\partial z^{2}}{\partial y}}\right)^{-1} \\
& \sin \varphi_{x}=\frac{\partial z}{\partial x}\left(\sqrt{1+\frac{\partial z^{2}}{\partial x}+\frac{\partial z^{2}}{\partial y}}\right)^{-1}
\end{aligned}
$$

The second integral $\mathrm{Int}_{12}$ is treated in a similar way:

$$
\begin{aligned}
& \operatorname{Int}_{12}(a, b)=\frac{1}{3 \pi}\left(1+\frac{\partial z^{2}}{\partial x}+\frac{\partial z^{2}}{\partial y}\right)^{-1} \\
& \times \int_{a}^{b}\left(1+\left(2-3 \cos \theta+\cos ^{3} \theta\right) M^{2}-2 \sin ^{3} \theta M-\cos ^{3} \theta\right) d \alpha \\
& M=\sin \alpha \frac{\partial z}{\partial x}+\cos \alpha \frac{\partial z}{\partial y}
\end{aligned}
$$

After simplification of the $\operatorname{Int}_{12}$ the integral difference $\Delta \operatorname{Int}_{12}$ from Eq. (8) can be solved using elementary functions:

$$
\begin{aligned}
\Delta \operatorname{Int}_{12} & =\left.\frac{2}{3 \pi}\left(X \sqrt{1-X^{2}}+\arcsin X\right)\right|_{-\beta} ^{\beta}, \\
X(\alpha) & =\sin \alpha \sin \varphi_{y}-\cos \alpha \sin \varphi_{x}
\end{aligned}
$$

Even though $\Delta \operatorname{Int}_{12}$ can be solved analytically, due to its complexity it can be barely used for further considerations and therefore it will be approximated using elementary functions. One of the possible approximations is the decomposition of the primitive function $\Delta \operatorname{Int}_{12}$ using Taylor series:

$$
\begin{aligned}
& \frac{1}{2}\left(X \sqrt{1-X^{2}}+\arcsin X\right)= \\
& X-\frac{1}{6} X^{3}-\frac{1}{40} X^{5}-\frac{1}{112} X^{7}+O\left(X^{9}\right)
\end{aligned}
$$

At small slope angles $X \ll 1$, hence for the characterization of the behaviour of the primitive function, the linear and cubic member of Eq. (13) are sufficient:

$$
\begin{aligned}
& \Delta \operatorname{Int}_{12}=\frac{8}{3 \pi} \sin \beta \sin \varphi_{y} \\
& \times\left(1-3 \kappa \cos ^{2} \beta+\kappa\left(3 \cos ^{2} \beta-\sin ^{2} \beta\right) \sin ^{2} \varphi_{y}+3 \kappa \cos ^{2} \beta \cos ^{2} \lambda\right)
\end{aligned}
$$

With $\kappa \approx 1 / 5.8$ and $\lambda$ as the angle between the $\mathrm{z}$-axis and the normal to the tangent plane:

$$
\begin{aligned}
\cos \lambda & =\left(\sqrt{1+\frac{\partial z^{2}}{\partial x}+\frac{\partial z^{2}}{\partial y}}\right)^{-1} \\
& =\sqrt{1-\sin ^{2} \varphi_{x}-\sin ^{2} \varphi_{y}}
\end{aligned}
$$

Finally, after evaluation of the integrals $\Delta \operatorname{Int}_{11}$ and $\Delta \operatorname{Int}_{12}$ the difference of the detector signals for Zone ${ }_{1 \_} 0^{\circ}$ can be determined as:

$$
\begin{aligned}
I_{2 y}-I_{1 y}= & \sigma(x, y) \sin \beta \sin \varphi_{y} \\
& \times\left(c_{1}+c_{2} \sin ^{2} \varphi_{y}+c_{3} \cos ^{2} \lambda\right)
\end{aligned}
$$

Whereas the coefficients $c_{1} \ldots c_{3}$ are the functions of the angle parameters $\beta$ and $\theta$. These functions are determined in Eqs. (8), (9) and (14). The sum of the detector signals depends only from Zone 3 and in case of $\theta=0^{\circ}$ is equal to the total emission yield at the irradiation point:

$$
I_{2 y}+I_{1 y}=\sigma(x, y)
$$

\section{ZONE 3}

Next the influence of the Zone 3 will be examined. Similarly to the calculation procedures for Zone 1, Zone 3 is also divided into elementary surfaces and the entire electron flow is computed as integral sum, see also Eq. (3):

$$
\begin{aligned}
\frac{I_{\text {Zone3 }}}{\sigma(x, y)}= & k_{1} \underbrace{\int_{-\pi}^{\pi} d \alpha \int_{0}^{\theta} f_{\text {Lam }}(\gamma, \alpha) \sin \gamma d \gamma}_{\operatorname{Int}_{31}(-\pi, \pi)} \\
& -k_{2} \underbrace{\int_{-\pi}^{\pi} d \alpha \int_{0}^{\theta} f_{\text {Lam }}^{2}(\gamma, \alpha) \sin \gamma d \gamma}_{\operatorname{Int}_{32}(-\pi, \pi)} .
\end{aligned}
$$

Where $\theta=$ const is the maximal angle, with which the emission electrons are absorbed by the electron gun. Both components $\operatorname{Int}_{31}(a, b)$ and $\operatorname{Int}_{32}(a, b)$ of the electron flow over Zone 3 in Eq. (18) are expressed as functions of the integration limits:

$$
\begin{gathered}
\operatorname{Int}_{31}(a, b)=\frac{1}{2 \pi}\left(\sqrt{1+\frac{\partial z^{2}}{\partial x}+\frac{\partial z^{2}}{\partial y}}\right)^{-1} \\
\times \int_{a}^{b}\left(M(\cos \theta \sin \theta-\theta)+\sin ^{2} \theta\right) d \alpha \\
M=\sin \alpha \frac{\partial z}{\partial x}+\cos \alpha \frac{\partial z}{\partial y}
\end{gathered}
$$




$$
\begin{aligned}
& \operatorname{Int}_{32}(a, b)=\frac{1}{3 \pi}\left(1+\frac{\partial z^{2}}{\partial x}+\frac{\partial z^{2}}{\partial y}\right)^{-1} \\
& \times \int_{a}^{b}\left(1+\left(2-3 \cos \theta+\cos ^{3} \theta\right) M^{2}-2 M \sin ^{3} \theta-\cos ^{3} \theta\right) d \alpha .
\end{aligned}
$$

Unlike Zones 1 and 2 the electron flow over Zone 3 is considered to be partly absorbed by the electron gun, which leads to a decrease of the sum of detector signals:

$$
\begin{aligned}
\frac{I_{1 y}+I_{2 y}}{\sigma(x, y)} & =1-\tau \frac{I_{\text {Zone3 }}}{\sigma(x, y)} \\
& =1-\tau k_{1} \operatorname{Int}_{31}(-\pi, \pi)+\tau k_{2} \operatorname{Int}_{32}(-\pi, \pi) \\
& =a_{0}-a_{1} \cos \lambda+a_{2} \cos ^{2} \lambda
\end{aligned}
$$

Here $\tau$ is the absorption coefficient of the electron gun and $a_{0}=1+\tau k_{2} \frac{1}{3}\left(2-3 \cos \theta+\cos ^{3} \theta\right), a_{1}=\tau k_{1} \sin ^{2} \theta, a_{2}=$ $\tau k_{2}\left(\cos \theta-\cos ^{3} \theta\right)$ are system specific constants.

Since the non-absorbed fraction of the emission electrons over Zone 3 is assumed to be split in half between the detectors 1 and 2 , it does not affect the difference of the detector signals. Therefore the entire difference of detector signals can be expressed as the signal difference over Zone Z $_{1} 0^{\circ}$ subtracting the absorbed electrons over the intersection of Zone 3 and Zone $_{1 \_0^{\circ}}$ :

$$
\begin{aligned}
& I_{2 y}-I_{1 y}=I_{2 y}\left(\text { Zone }_{1 \_0^{\circ}}\right)-I_{1 y}\left(\text { Zone }_{1 \_0^{\circ}}\right) \\
& \quad+I_{1 y}\left(\text { Zone }_{3} \cap \text { Zone }_{1 \_0^{\circ}}\right)-I_{2 y}\left(\text { Zone }_{3} \cap \text { Zone }_{1 \_0^{\circ}}\right) .
\end{aligned}
$$

For the difference $\Delta I_{Z_{\text {Zne }}}=I_{1 y}\left(\right.$ Zone $_{3} \cap$ Zone $\left._{1 \_0^{\circ}}\right)-$ $I_{2 y}\left(\right.$ Zone $_{3} \cap$ Zone $\left._{1 \_0^{\circ}}\right)$ the following equation is valid:

$$
\begin{aligned}
\frac{\Delta I_{\text {Zone3 }}}{\sigma(x, y)}= & k_{1} \underbrace{\left(\operatorname{Int}_{31}(-\beta, \beta)-\operatorname{Int}_{31}(\pi-\beta, \pi+\beta)\right)}_{\Delta \operatorname{Int}_{31}} \\
& -k_{2} \underbrace{\left(\operatorname{Int}_{32}(-\beta, \beta)-\operatorname{Int}_{32}(\pi-\beta, \pi+\beta)\right)}_{\Delta \operatorname{Int}_{32}} .
\end{aligned}
$$

The integration of summands leads to:

$$
\begin{aligned}
\Delta \operatorname{Int}_{31} & =-\frac{1}{\pi}(2 \theta-\sin (2 \theta)) \sin \beta \sin \varphi_{y}, \\
\Delta \operatorname{Int}_{32} & =-\frac{8}{3 \pi} \sin ^{3} \theta \sin \beta \sin \varphi_{y} \cos \lambda .
\end{aligned}
$$

Thus the signal relation with consideration of the efficiency of the detector system can be represented as follows:

$$
\begin{aligned}
& k_{y}(x, y)=\frac{I_{2 y}-I_{1 y}}{I_{2 y}+I_{1 y}} \\
& =\sin \varphi_{y} \sin \beta \frac{b_{1}+b_{2} \sin ^{2} \varphi_{y}+b_{3} \cos ^{2} \lambda+b_{4} \cos \lambda}{a_{0}-a_{1} \cos \lambda+a_{2} \cos ^{2} \lambda}
\end{aligned}
$$

The presented signal relation refers to detectors, aligned along the $y$-axis. The computation for the detectors along the $\mathrm{x}$-axis is similar, hence:

$$
\begin{aligned}
& k_{x}(x, y)=\frac{I_{2 x}-I_{1 x}}{I_{2 x}+I_{1 x}} \\
& =\sin \varphi_{x} \sin \beta \frac{b_{1}+b_{2} \sin ^{2} \varphi_{x}+b_{3} \cos ^{2} \lambda+b_{4} \cos \lambda}{a_{0}-a_{1} \cos \lambda+a_{2} \cos ^{2} \lambda}
\end{aligned}
$$

To complete modelling of the signal, the influence of the surface must be considered, because the electrons with a small emission angle are partly absorbed by the surface. For the quantitative evaluation of the unregistered fraction of the emitted electrons the maximal directly registerable angle $\phi_{\max }$ should be determined. This parameter defines the maximal angle, at which the electrons are still not absorbed through the surface of the specimen. The evaluation of this angle has to be made for each measuring point $(x, y)$, and for each azimuthal emission angle $\alpha$ (see Figure 4(b) ). Respecting zone splitting, the unregistered emission fraction can be calculated as follows:

$$
\begin{aligned}
\xi\left(x, y, \beta_{1}, \beta_{2}\right)=\frac{k_{1}}{2 \pi} \int_{\beta_{1}}^{\beta_{2}} \cos ^{2} \phi_{\max }(\alpha) d \alpha \\
\quad-\frac{k_{2}}{3 \pi} \int_{\beta_{1}}^{\beta_{2}} \cos ^{3} \phi_{\max }(\alpha) d \alpha
\end{aligned}
$$

whereby $\beta_{1}$ and $\beta_{2}$ describe the lateral angle-borders of Zone 1 .

Finally, the signal relation for detectors, aligned along the yaxis, takes the following form:

$$
\begin{aligned}
k y= & \frac{I_{2 y}(x, y)-I_{1 y}(x, y)}{I_{2 y}(x, y)+I_{1 y}(x, y)} \\
= & \frac{1}{\operatorname{Den}(\lambda)-\tau_{\mathrm{sp}} \xi(-\pi, \pi)}\left[\sin \psi_{y} \operatorname{Nom}\left(\psi_{y}, \lambda\right) \sin \beta\right. \\
& \left.-\tau\left(\xi\left(-\beta+\frac{\pi}{2}, \beta+\frac{\pi}{2}\right)-\xi\left(-\beta-\frac{\pi}{2}, \beta-\frac{\pi}{2}\right)\right)\right] .
\end{aligned}
$$

Here, $I_{1 y}, I_{2 y}$ are the measured detector signals, and $\tau_{\mathrm{sp}}$ and $\tau$ are the absorption coefficients of the specimen and of the electron gun respectively,

$$
\begin{aligned}
\lambda & =\arccos \sqrt{1-\sin ^{2} \psi_{x}-\sin ^{2} \psi_{y}}, \\
\sin \psi_{x} & =\frac{\partial z}{\partial x}\left(\sqrt{1+\frac{\partial z^{2}}{\partial x}+\frac{\partial z^{2}}{\partial y}}\right)^{-1}, \\
\sin \psi_{y} & =\frac{\partial z}{\partial y}\left(\sqrt{1+\frac{\partial z^{2}}{\partial x}+\frac{\partial z^{2}}{\partial y}}\right)^{-1} .
\end{aligned}
$$

Further components of the Eq. (29) describe the dependence of the signal relation on the local inclination angle of the specimen:

$$
\begin{aligned}
\operatorname{Nom}\left(\psi_{y}, \lambda\right) & =b_{1}+b_{2} \sin ^{2} \psi_{y}+b_{3} \cos ^{2} \lambda+b_{4} \cos \lambda \\
\operatorname{Den}(\lambda) & =a_{0}-a_{1} \cos \lambda+a_{2} \cos ^{2} \lambda
\end{aligned}
$$

where the coefficients $a_{0} \ldots a_{2}$ and $b_{1} \ldots b_{4}$ are presented as functions of the previously mentioned system parameters $\theta$ and $\beta$. Here one can see that Eqs. (29)-(30) correspond to Eq. (1) mentioned the in the beginning.

The system parameters $\theta$ and $\beta$ depend on the hardware constellation of SEM, like working distance, initiating electron energy etc., and are determined by using the method of the maximum likelihood estimation on the calibration steel ball with the diameter of $1.2 \mathrm{~mm}$. Hereby the height values are measured indirectly over the detector signal relations. For this reason the target function refers to the measured and modelled 
signal relations, see Eq. (29):

$$
\begin{aligned}
& Z F=\sum_{i, j=1}^{N, M}\left[k_{x i j}-k_{x}\left(x_{i}, y_{j} ; a_{0} . . a_{2}, b_{1} . . b_{4}\right)\right]^{2} \\
& +\sum_{i, j=1}^{N, M}\left[k_{y i j}-k_{y}\left(x_{i}, y_{j} ; a_{0} . . a_{2}, b_{1} . . b_{4}\right)\right]^{2} \rightarrow \min _{a_{0} . . a_{2}, b_{1} . . b_{4}}
\end{aligned}
$$

with $k_{x}, k_{y}$ are model based and $k_{x i j}, k_{y i j}$ are experimentally obtained signal relations

The unknown coefficients $a_{0} \ldots a_{2}$ and $b_{1} \ldots b_{4}$ are based on: $k_{1}, k_{2}$ are the weight coefficients of the angular distribution in Eq. (2), $\tau, \tau_{\mathrm{sp}}$ are the absorption coefficients and $\theta, \beta$ are the angle parameters for zone splitting.

The determination of the signal relations is to be done along the $y$ - and the $x$-axis. So, for each point $(x, y)$ their exist two equations, connecting the detector signals, surface function $z(x, y)$ and the partial derivatives $z_{x}^{\prime}$ and $z_{y}^{\prime}$. Unfortunately, $z(x, y)$ is unknown, therefore the reconstruction algorithm takes form of the iterative procedure. At the 0-Iteration the surface is considered to be constant: $z(x, y)=0$ and the partial derivatives are computed using Eq. (29):

1. Numeric integration of the partial derivations, which results in the new approximation of the real surface.

2. Based on the in step 2 reconstructed surface, the unregistered fractions of the emitted electrons are re-computed using Eq. (28). These fractions act as basement for the reevaluation of the partial derivatives with Eq. (29).

3. If the difference between the partial derivatives $z_{x}^{\prime}$ and $z_{y}^{\prime}$, computed in two sequential iterations is smaller than the given threshold, the algorithm will be terminated, otherwise the iteration will be repeated.

Usually the computed surface converges very fast against the real one.

\section{EXPERIMENTS}

This iterative procedure was used to examine the accuracy of measurement with a steel ball sample of $1.2 \mathrm{~mm}$ diameter. As Figure 7 shows, for small slope angles the 3D reconstruction fits a nearly perfect spherical shape. After reaching a slope angle of approximately $45^{\circ}$, the deviation in the height increases strongly.

This circumstance can be explained by the non unique correspondence between the signal relation $k_{x}=\left(I_{2 x}-I_{1 x}\right)\left(I_{2 x}+I_{1 x}\right)^{-1}$ and the local slope angle, as it is shown in Figure 7. Here the ball is positioned in the centre of the image, so the inclination decreases monotonically along the marked lines. In contrast to the inclination, the signal relation $k_{x}$ can be distinguished as a monotonically decreasing function only for small slope angles.

After reaching the turning point at around $45^{\circ}$ the signal relation $k_{x}$ changes its orientation. The angle of this turning point

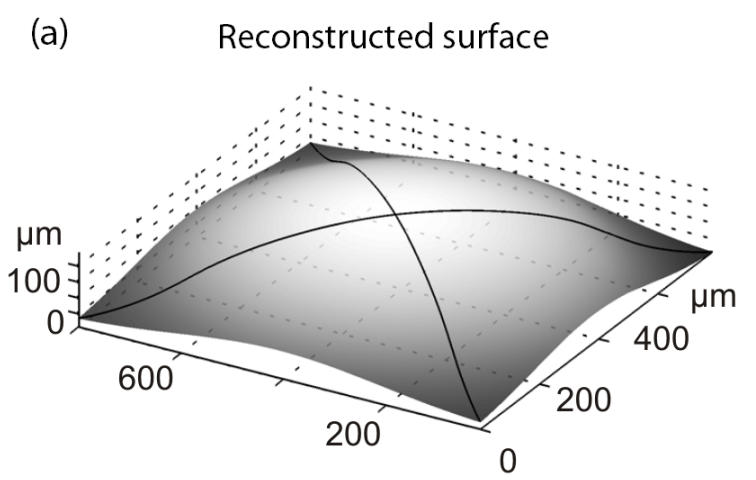

(b)

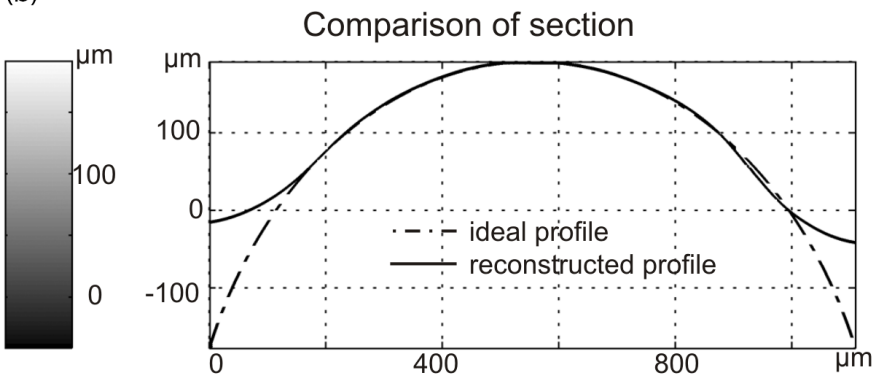

FIG. 7 3D-reconstruction of a steel ball: (a) total reconstruction result, (b) marked diagonal profile and corresponding profile.
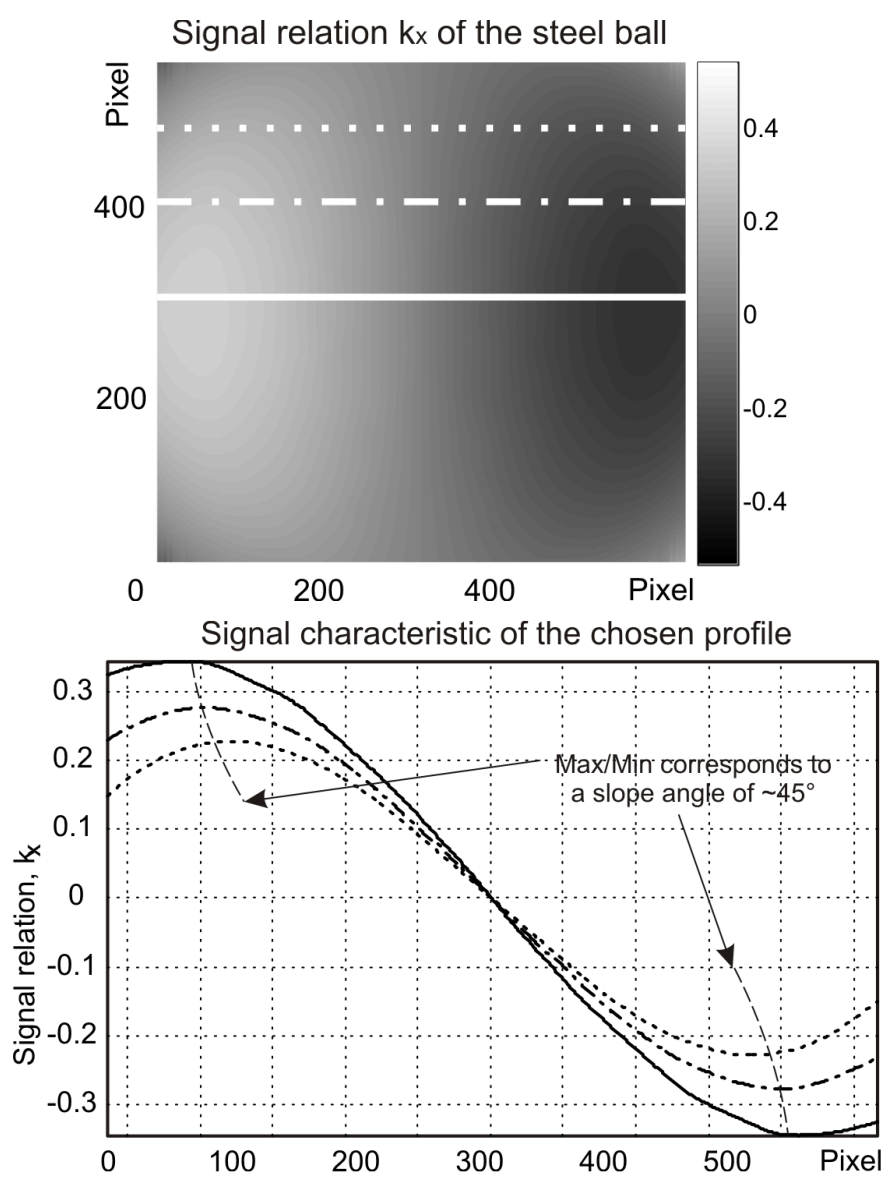

FIC. 8 Calculated signal relation $k_{x}$ of the steel ball. 


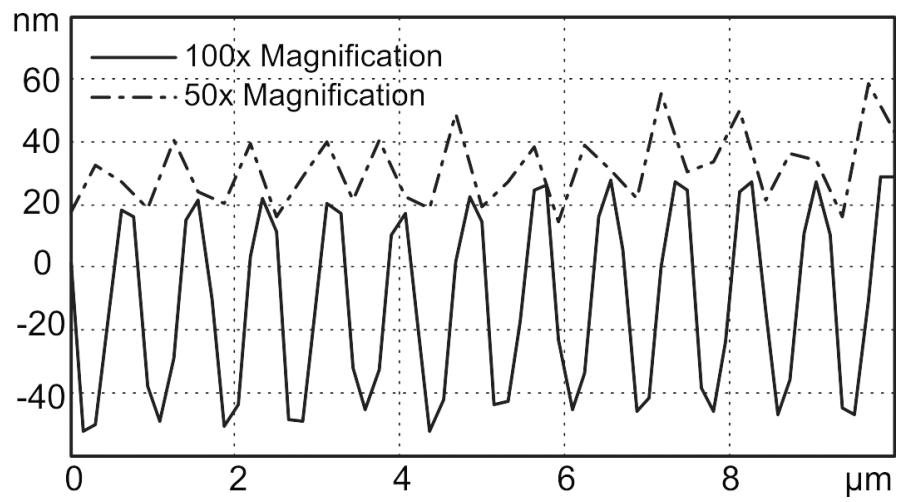

FIG. 9 Measurement of a holographic diffractive grating with a confocal microscope.

depends on the SEM and can be increased significantly by taking proper precautions like shielding the electron gun [6]. While reconstructing, the slope is derived out of the signal relation where an ambiguity of the determination of the surface gradient occurs and smallest possible slope will be chosen.

For slope angles between $0^{\circ}$ and $45^{\circ}$ the deviation is less than $5 \mu \mathrm{m}$. Though the deviation of the measuring results is relatively large, it is crucial that the reconstruction algorithm is only connected to the slope and therefore linearly dependent on the dimension of the sample. If, for example, a steel ball of $10 \mu \mathrm{m}$ diameter is measured an accuracy of approximately $50 \mathrm{~nm}$ can be expected.

The advantages of the SEM over optical measuring methods will be highlighted with a holographic diffractive grating of $830 \mathrm{~nm}$. Because of the physical resolution restriction of about $200 \mathrm{~nm}$, the optical measuring devices are at their limit although the grating period length is relatively large. While the sine wave is identifiable with $100 \times$ magnification, it can't be measured with $50 \times$ magnification due to poor lateral resolution of $300 \mathrm{~nm}$ per pixel (see Figure 9).

Since tactile measuring methods might damage the surface they also cannot be used for collecting topography data. Though the atomic force microscope (AFM) in non-contact mode is able to fulfil the measuring task, the time effort is so tremendous that measuring objects with higher complexity like holograms is not feasible in a reasonable amount of time.

Figure 10 shows the SEM and AFM measurement results. Due to the homogeneous character of the grating surface, an exact localization of the measuring position and therefore the comparison between the two different methods is barely possible. Nonetheless a structural height of $60 \mathrm{~nm}$ to $80 \mathrm{~nm}$ is noticeable in both, the SEM and the AFM, measurements. While it took the SEM (DSM 940A, 2 ET-detectors, Diss5 Point Electronics imaging acquisition unit) about 10 minutes, the AFM (SIS NANOstation II) required 6 hours. In addition the SEM can be operated interactively, while it is nearly impossible to do repetitive measurements with the AFM after changing the sample's position.

It is possible to measure even more complicated structures like a hologram (see Figure 11). This hologram has dimensions of

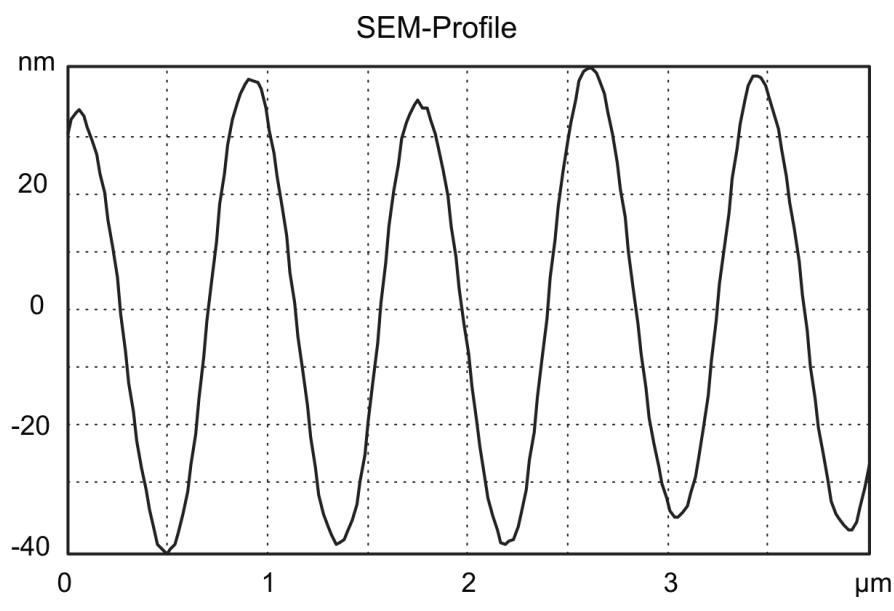

AFM-Profile

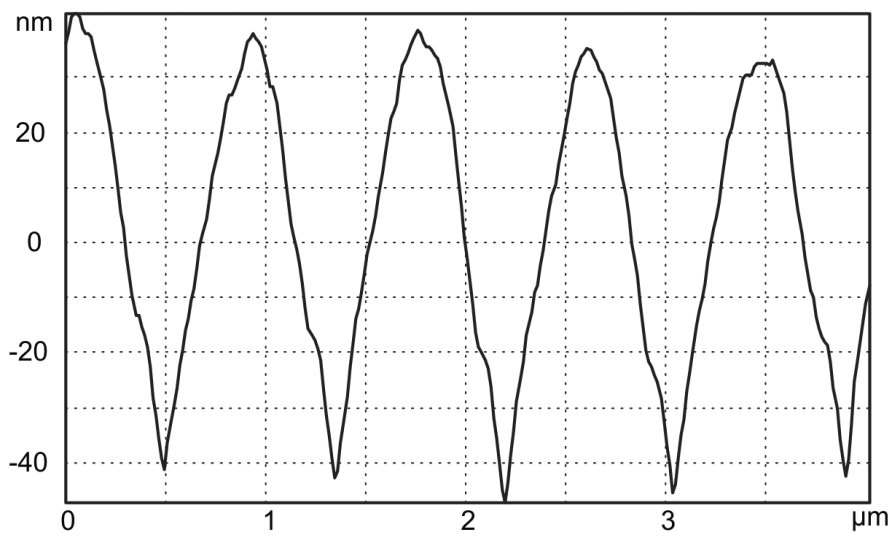

FIG. 10 Comparison between SEM profile and AFM profile. Measurement of a holographic grating wtih $830 \mathrm{~nm}$ wavelength.

$8000 \times 8000$ pixels and different wavelength and amplitudes. As it can be seen in the detailed pictures the reconstruction algorithm has also the ability to manage more complicated structures.

\section{CONCLUSION}

The algorithm was implemented and experimentally tested regarding its accuracy. The presented results prove that the modified SEM method is suitable to use up to the inclination angle of $45^{\circ}$ for investigations of surfaces and flat structures to obtain very high lateral and vertical resolution data (up to $30 \mathrm{~nm}$ ). This method gives the possibility to measure DOEs with a reasonable effort of time, where optical and tactile measuring devices fail. For the measurement of structures with a slope angle above $45^{\circ}$ an improvement of the setup is needed. This can be fulfilled by optimizing the field distribution near the sample and by reducing the influence of the electron gun.

\section{ACKNOWLED GEMENTS}

The presented work and results was financially supported by the German Research Foundation (DFG) under grants RE1488/11-3 and FA887/1-1. 
SEM-Image(total), Detector $1,0^{\circ}$

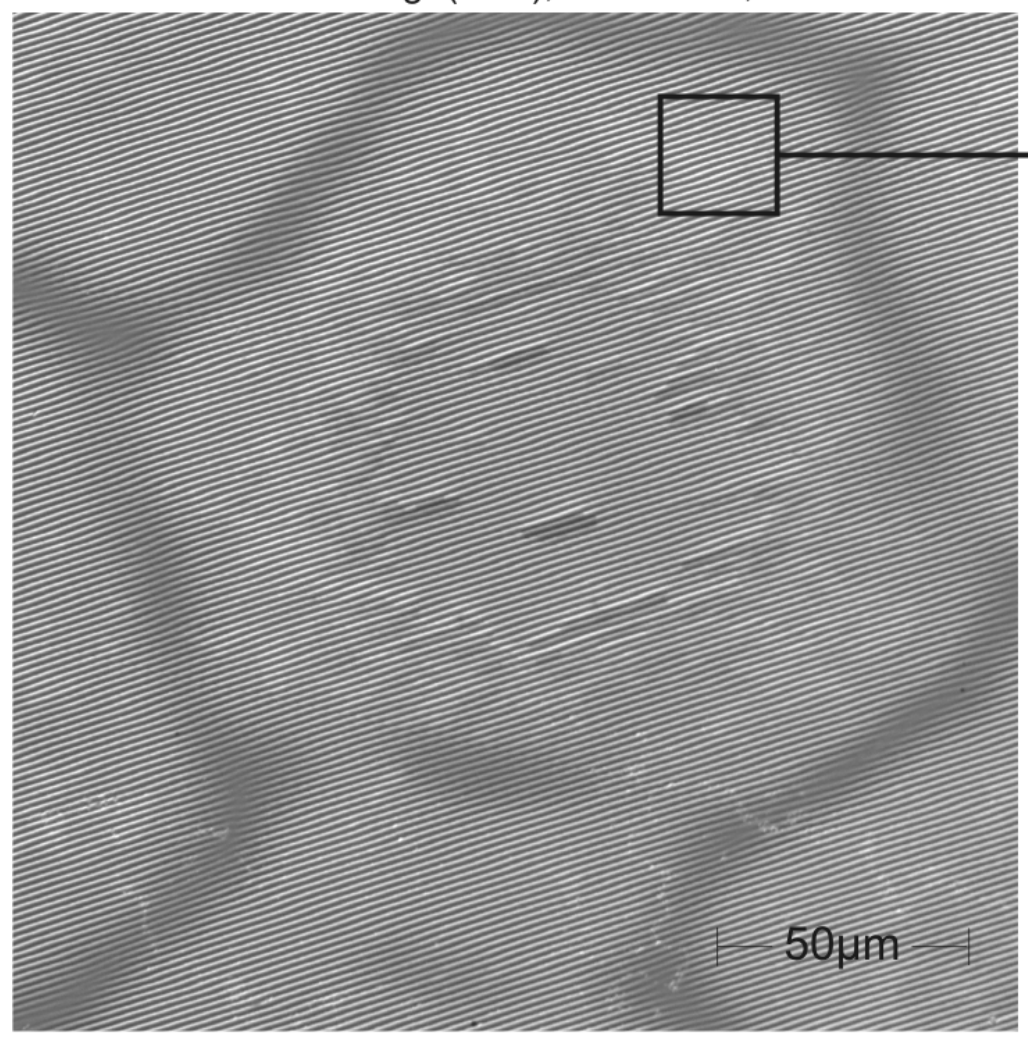

Magnification 200x, WD=13mm, HV=3kV
SEM-Image (detail)

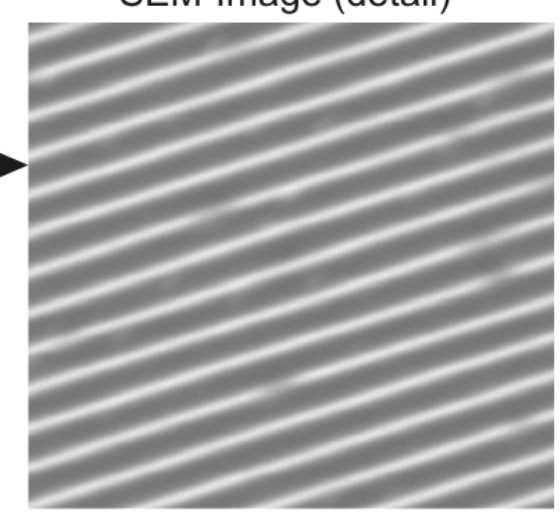

Reconstruction result

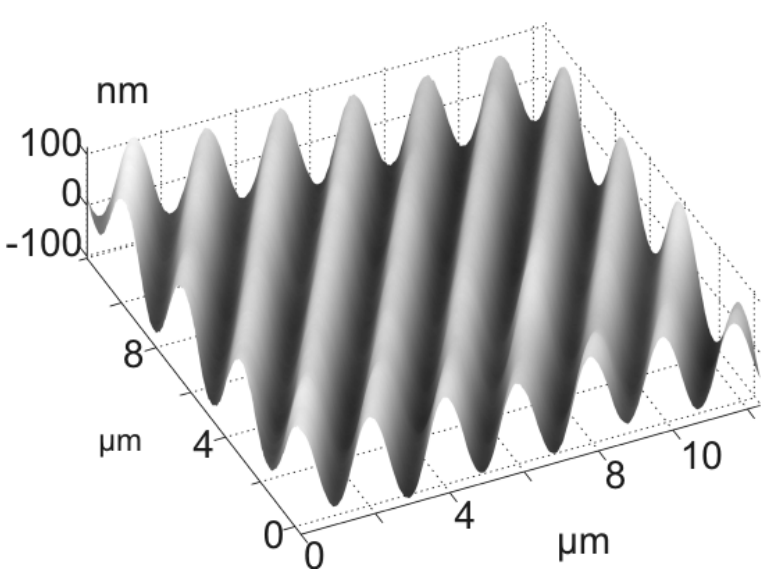

FIG. 11 Picture of a hologram $8000 \times 8000$ pixels. On the right side, a more detailed SEM picture and its 3 D reconstruction.

\section{References}

[1] L. Reimer, Image Formation in Low-Voltage Scanning Electron Microscopy (SPIE Optical Engineering Press, Bellingham, 1993).

[2] W. Beil, and I. C. Carlsen, "Surface reconstruction from stereoscopy and shape from shading" in SEM images" Mach. Vision Appl. 4, 271-285 (1991).

[3] J. Paluszynski, and W. Slowko, "Surface reconstruction with the photometric method in SEM" Vacuum 78, 533-537 (2005).

[4] W. Slowko, and W. Drzazga, "Detection of secondary electrons in a retarding electric field" Vacuum 63, 463-467 (2001).

[5] C. Tollkamp, Rekonstruktion des oberflächenprofils in einem
Raster-Elektronenmikroskops mit einem Zwei-Detektor System (Dissertation, Universität Münster, 1984).

[6] M. Kässens, and L. Reimer, "Contrast effects using a 2-detector system in low voltage scanning electron microscopy" J. Microsc.Oxford 181, 277-285 (1996).

[7] J. Cazaux, "Recent developments and new strategies in scanning electron microscopy" J. Microsc.-0xford 217, 16-35 (2005).

[8] I. Müllerova, and I. Konvalina, "Collection of secondary electrons in scanning electron microscopes" J. Microsc.-0xford 236, 203-210 (2009). 